\title{
Simulation of Jet Noise with OVERFLOW CFD Code and Kirchhoff Surface Integral
}

\author{
M. Kandula \\ Dynacs Inc., Kennedy Space Center, FL \\ R. Caimi \\ NASA Kennedy Space Center, FL
}

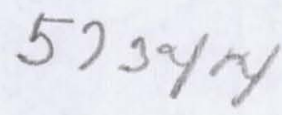

\begin{abstract}
An acoustic prediction capability for supersonic axisymmetric jets was developed on the basis of OVERFLOW Navier-Stokes CFD (Computational Fluid Dynamics) code of NASA Langley Research Center. Reynolds-averaged turbulent stresses in the flow field are modeled with the aid of Spalart-Allmaras one-equation turbulence model. Appropriate acoustic and outflow boundary conditions were implemented to compute timedependent acoustic pressure in the nonlinear source-field. Based on the specification of acoustic pressure, its temporal and normal derivatives on the Kirchhoff surface, the near-field and the far-field sound pressure levels are computed via Kirchhoff surface integral, with the Kirchhoff surface chosen to enclose the nonlinear sound source region described by the CFD code. The methods are validated by a comparison of the predictions of sound pressure levels with the available data (Seiner et al., 1992) for an axisymmetric turbulent supersonic (Mach 2) perfectly expanded jet.
\end{abstract}

\section{$\underline{\text { Nomenclature }}$}

$c=$ sound speed, $\sqrt{\gamma g_{c} R_{u} T / W}, \mathrm{ft} / \mathrm{s}$

$d_{j}=$ nozzle exit diameter, $\mathrm{ft}$

$F=$ total thrust, $\mathrm{lbf}$

$f=$ frequency, $\mathrm{Hz}$

$g_{c}=$ gravitational constant, $\left(\mathrm{lbm}-\mathrm{ft} / \mathrm{lbf} . \mathrm{s}^{2}\right)$

$I$ = specific impulse (sec)

$M=$ Mach number, $V / c$

$m=$ instability mode

$p=$ static pressure, $\mathrm{lbf} / \mathrm{ft}^{2}$

$p^{\prime}=$ acoustic pressure disturbance (instantaneous

pressure), $p-p_{m}, \mathrm{lbf} / \mathrm{ft}^{2}$

$Q=$ vector of disturbance variables

$\mathrm{Re}=$ Reynolds number,$\quad \rho u_{j} d_{j} / \mu$

$R_{u}=$ universal gas constant, $\mathrm{lbf} . \mathrm{ft} /(\mathrm{lbm}-\mathrm{mole} \mathrm{R})$

$r=$ radial distance

$r_{j}=$ nozzle exit radius, $\mathrm{ft}$

$S t=$ Strouhal number, $f d_{j} / u_{j}$

$T$ = temperature, $\mathrm{R}$
$V=$ velocity, $\mathrm{m} / \mathrm{s}$

$u=$ axial velocity, $\mathrm{ft} / \mathrm{s}$

$u_{c}=$ jet center-line velocity at exit, $\mathrm{ft} / \mathrm{s}$

$w=$ radial velocity, $\mathrm{ft} / \mathrm{s}$

$W=$ molecular weight, $\mathrm{lbm} / \mathrm{lbm}$-mole

$x=$ axial distance from the nozzle exit, $\mathrm{ft}$

$z=$ radial distance from jet axis, $\mathrm{ft}$

\section{Greek Symbols}

$\mu$ = dynamic viscosity, $\mathrm{lbm} . \mathrm{ft} / \mathrm{s}$

$\rho=$ density, $\mathrm{lbm} / \mathrm{ft}^{3}$

$\gamma=$ isentropic exponent

$\omega=$ circular frequency, radians $/ \mathrm{s}$

$\psi=$ angle from jet axis, deg

\section{$\underline{\text { Subscripts }}$}

$c=$ centerline

$e=$ nozzle exit

$j=$ jet

$t=$ stagnation or total, or nozzle throat

$\infty=$ ambient

\section{$\underline{\text { Superscripts }}$}

prime : disturbance from the mean value

\section{Introduction}

Acoustic loads in a launch vehicle environment represent a principal source for inducing structural vibration and may be critical to the proper functioning of vehicle components and ground support structures and equipment in the immediate vicinity of the launch pad. A knowledge of acoustic loads, including the overall sound pressure level (OASPL), sound pressure level (SPL) spectrum, and the distribution (or correlation) of surface acoustic loads, is necessary to provide the input for vibroacoustic analysis and evaluation of structural integrity. In the design of launch vehicles, it is highly desirable that data on acoustic loads (near-field and farfield noise levels) be generated both analytically and from testing of small-scale and full-scale models. Since fullscale acoustic and vibration testing is often cost prohibitive, the option of small scale testing combined 
with analysis methods remains as a practical alternative. Accurate characterization of acoustic loads on launch pad structures thus proves to be a formidable challenge

Noise from subsonic jets is mainly due to turbulent mixing, comprising the contributions of large-scale and fine-scale structures (Lighthill ${ }^{1,2}$ ). The turbulent mixing noise is mainly broadband. In perfectly expanded supersonic jets (nozzle exit plane pressure equals the ambient pressure), the large-scale mixing noise manifests itself primarily as Mach wave radiation, caused by the supersonic convection of turbulent eddies with respect to the ambient fluid. In imperfectly expanded supersonic jets, additional noise is generated on account of broadband shock noise and screech tones.

The prediction of noise generation and propagation from exhaust jets by computational methods entails certain numerical requirements $\left(\mathrm{Tam}^{3}\right)$. Although the existing CFD codes can predict the noise generation in the nonlinear source-field, their application to acoustic predictions for obtaining near-field and far-field sound levels results in inaccuracies due to numerical dispersion and dissipation over long propagation distances. Specialized Computational Aeroacoustics (CAA) methods were developed to resolve these numerical issues satisfactorily (Tam and $\mathrm{Webb}^{4}, \mathrm{Lele}^{5}$ ). These computational methods for direct calculation of noise in the near- and the far-field, however, require prohibitively large computational resources.

This situation motivated in recent years the application of CFD codes to model the source region (containing the sound sources), with the near- and farfield sound predicted by either Lighthill's acoustic analogy (Lighthill ${ }^{1,2}$ ) or the Kirchhoff surface integral (Kirchhoff6). In Lighthill's acoustic analogy the NavierStokes equations are rearranged in wave-like form with Lighthill's stress tensor acting as the source term, and the near-field and far-field sound is obtained via a volume integral. This volume integral again requires large computational resources. On the other hand, the Kirchhoff method requires only a surface integral, with the Kirchhoff surface enclosing the nonlinear source region, and is chosen in a region where the linear wave equation is valid. Additionally, the Kirchhoff method does not suffer dissipation and dispersion errors when the nearfield and far-field sounds are directly calculated with the CFD codes. By restricting the use of CFD methods to the nonlinear near-field region for source identification and employing Kirchhoff integration for the linear region, computational requirements are greatly reduced.

The ability to accurately predict the turbulent mixing noise sources remains as a key requirement in the overall acoustic analysis. Both the large-scale turbulence structures (instability waves) and the fine-scale turbulence represent the mixing noise sources. The large turbulence structures are responsible for Mach wave emission that is directional, and the fine-scale turbulence mixing noise is fairly uniform independent of direction. In the past the two-equation $k-\varepsilon$ turbulence model is generally used to model the sound sources. However, this model entails large computational requirements (two additional equations) and is often plagued with convergence problems in flows with shock waves and strong gradients. Furthermore, the universal constants in the turbulence model are often altered to fit the theory with the acoustic measurements. On the other hand, the simple algebraic mixing length type turbulence model cannot accurately describe the growth of the mixing layers. Thus a oneequation turbulence model seems to be an attractive choice for engineering applications. This model generally results in faster convergence and requires less computational resources.

The objective of this investigation is to predict supersonic jet acoustics on the basis of CFD/ Kirchhoff formulation in conjunction with a one-equation turbulence model. This report summarizes the development of a computational procedure in which the OVERFLOW CFD Navier-Stokes code (Buning et al. ${ }^{7}$ ) predicts the nonlinear source-field within the jet and application of Kirchhoff surface integral for acoustic radiation.

\section{Analysis}

This method of analysis is based on the application of a CFD code for identifying the noise sources in the source-field and Kirchhoff surface integral for the propagation of sound radiation to the near-field and the far-field.

\subsection{CFD Analysis}

\subsubsection{Numerical Algorithm}

For the CFD analysis, the OVERFLOW code (Buning et al., 1998) of NASA Langley is used for computing the instantaneous flowfield. This code solves the three-dimensional compressible turbulent flow Navier-Stokes equations in generalized coordinates. The one-equation turbulence model of Spalart-Allmaras ${ }^{8}$ is considered.

\subsubsection{Boundary Conditions}

To simulate the exterior (infinite boundary) acoustic problem, we need to prescribe outflow and radiation boundary conditions at the boundaries of a finite computational domain. These boundary conditions must be sufficiently transparent to the outgoing disturbances so that they exit the computational domain without significant (nonphysical) reflections. It is assumed that the boundaries are sufficiently far from the sources.

The OVERFLOW code, in its present form, is designed primarily for the prediction of steady or 
unsteady turbulent flowfields and used widely to model aerodynamic flows. It does not however provide the necessary time-dependent boundary conditions for handling the reflection-free acoustic propagation. Therefore appropriate modifications to the code were made to provide a set of time-dependent reflection-free boundary conditions (includes periodic inflow, outflow, and radiation).

\section{a. Inflow Boundary Conditions.}

At the supersonic inflow, all data are specified, such that all time variations of the characteristic variables are set to zero because all waves are incoming waves.

\section{b. Outflow Boundary Conditions.}

In general at the outflow boundary, the outgoing disturbances consist of acoustic, entropy, and vorticity waves. Here we follow Thompson's approach (Thompson') based on one-dimensional characteristics analysis (Riemann variables). The amplitude of the outward propagating waves at the boundary are specified entirely from the solution within the computational domain, while the amplitude of the inward propagating waves is prescribed at the boundary. For details on the method, the original reference should be consulted.

\section{c. Radiation Boundary Condition.}

At boundaries where there exist only outgoing acoustic waves, Tam and $\mathrm{Webb}^{4}$ developed a set of radiation boundary conditions based on an asymptotic analysis (valid for large distances). These boundary conditions, originally formulated in a spherical coordinate system, when applied to a cylindrical coordinate system, are given by (Hixon et al. ${ }^{10}$ )

$$
Q_{t}^{\prime}=-V\left[\frac{x}{R} Q_{x}^{\prime}+\frac{r}{R} Q_{r}^{\prime}+\frac{Q^{\prime}}{R}\right]
$$

where

$$
\begin{aligned}
& Q^{\prime}=\left[\rho^{\prime}, u^{\prime}, w^{\prime}, p^{\prime}\right]^{T}, \\
& V=c_{0}\left[\frac{x}{R} M+\sqrt{1-\left(\frac{r}{R} M\right)^{2}}\right], \\
& R=\sqrt{x^{2}+r^{2}}
\end{aligned}
$$

In the above equations, $Q^{\prime}$ is the vector of disturbance variables, $x$ the axial distance from the origin, and $r$ the radial distance from the jet axis. The subscripts on $Q^{\prime}$ indicate partial derivatives.

\subsection{Kirchhoff Code}

For the acoustic radiation, the Kirchhoff code YORICK developed by Pilon and Lyrintzis ${ }^{11}$ of Purdue University was considered. The Kirchhoff surface, enclosing the nonlinear source region, is chosen in a region where the linear wave equation is valid. Outside the Kirchhoff surface, the flow is governed by the linear equation

$$
\nabla^{2} p^{\prime}-\frac{1}{c_{\infty}^{2}}\left(\frac{\partial}{\partial t}+u_{\infty} \frac{\partial}{\partial x}\right)^{2} p^{\prime}=0
$$

where $u_{\infty}$ is the freestream (ambient) velocity. In the special case of stationary ambient $\left(u_{\infty}=0\right)$, it reduces to the simple wave equation. A solution to the pressure field can be expressed by the surface integrals as (Morino and Tseng ${ }^{12}$, Lyrintzis \& Mankbadi ${ }^{13}$ )

$$
\begin{aligned}
& p^{\prime}(x, y, z, t)=-\frac{1}{4 \pi} \\
& {\left[\begin{array}{l}
\frac{p^{\prime}}{r_{0}^{2}} \frac{\partial r_{0}}{\partial n_{0}}+\frac{1}{r_{0}} \frac{\partial p^{\prime}}{\partial n_{0}}+ \\
\frac{1}{c_{\infty} r_{0} \beta^{2}} \frac{\partial p^{\prime}}{\partial t}\left(\frac{\partial r_{0}}{\partial n_{0}}-M_{\infty} \frac{\partial x_{0}^{\prime}}{\partial n_{0}}\right)
\end{array}\right]_{\tau} d S_{0}}
\end{aligned}
$$

where

$$
\begin{aligned}
& r=\left\{\left(x-x^{\prime}\right)^{2}+\beta^{2}\left[\left(y-y^{\prime}\right)^{2}+\left(z-z^{\prime}\right)^{2}\right]\right\}^{2} \\
& \tau=\frac{\left[r_{0}-M_{\infty}\left(x-x^{\prime}\right)\right]}{c_{\infty} \beta^{2}}, \quad \beta=\left(1-M_{\infty}^{2}\right)^{1 / 2}
\end{aligned}
$$

In the above equations, $M_{\infty}$ is the freestream Mach number, $c_{\infty}$ the speed of sound in the freestream, the prime a point on the Kirchhoff surface, $\tau$ the retarded time $\tau=t-t^{\prime}, n_{0}$ the outward normal to the Kirchhoff surface $S_{0}$. Thus the pressure at any instant in the region outside the Kirchhoff surface can be expressed in terms of the information prescribed on the Kirchhoff surface. The required data on pressure, and its normal and temporal derivatives on the Kirchhoff surface are taken from the CFD solution.

\section{Validation Test Case}

The CFD/Kirchhoff methodology formulated above is tested by comparing the predictions with the wellknown acoustic test data of Seiner et al. ${ }^{14}$ for Mach 2 perfectly expanded supersonic jets of air. The nozzle exit radius is $0.04572 \mathrm{~m}$. Although the data cover a wide 
range of jet total temperature (using propane burners), detailed validation is performed here for a jet total temperature of 755 kelvin $(\mathrm{K})$. Validation at other jet total temperatures will be reported in a later report. Table 1 shows the data at the nozzle exit. The jet exit velocity is 822 meters per second $(\mathrm{m} / \mathrm{s})$, and exhausts into quiescent surroundings at a temperature of $280 \mathrm{~K}$. Figure 1 shows the location of the far-field measurements of the OASPL with which comparisons with the CFD results are reported.

\section{Numerical solution}

\subsection{CFD Solution}

\subsubsection{Axisymmetric Grid}

For the CFD computations, an axisymmetric grid of size $200 \times 100 \times 3$ (200 grid points in the axial direction, 100 grid points in the radial direction, and 3 in the azimuthal plane) is considered (figure 2). A grid length of 60 jet radii and a grid radius of 10 radii are considered. The grid is clustered in the radial direction near the nozzle wall to resolve the shear layer. Because of the perfectly expanded condition, uniform grid is considered adequate in the axial direction.

\subsubsection{Flowfield Solution}

At the inlet, the flow variables are specified based on the nozzle exit conditions. In addition, a periodic disturbance (axisymmetric mode) of a single frequency is also prescribed as follows:

$$
u^{\prime}(z)=A \sin (\omega t) \exp \left[-\ln (2)\left(\frac{z-h}{b}\right)^{2}\right]
$$

where $\omega$ is the circular frequency of the disturbance and $A$ the amplitude of disturbance. Based on the work of Mankbadi et al. ${ }^{15}$, values of $b=0.1$ and $h=0.78361$ are considered. The amplitude $A$ is taken to be of the order of 0.01. A value of $S t=0.2$ (based on typical peak SPL frequency for supersonic jets) is considered here for obtaining the disturbance frequency $\omega$.

Appropriate time-dependent boundary conditions were applied to ensure reflection-free boundaries. An outflow boundary condition of Thompson type (Thompson 1990) is applied at the outflow boundary, which maintains the mean static pressure at the ambient value. An acoustic radiation boundary condition of Tam and Webb ${ }^{4}$ is applied at the lateral boundary.

The CFD solution converges after about 8,000 timestep iterations (the code is run in a time-accurate manner) before a periodic state is established. An initial steadystate solution (based on local time-stepping, requiring about 600 iterations for convergence) has served as the starting solution for the time-accurate run. The solution is obtained on an IRIX workstation (SGI Indigo machine). About 15 hours of computing time are required for the solution to achieve a periodic state.

\subsection{Acoustic Solution}

After a periodic state has been established, the appropriate data from CFD, corresponding to a cycle, is communicated to the Kirchhoff code. The Kirchhoff surface is a cylindrical surface coinciding with a grid line. The radius of the Kirchhoff surface is taken as about 6 radii from the jet axis. Only the lateral surface of the cylinder is taken into account, and the cylinder ends are neglected due to the effects of nonlinearities. The data to be specified on the Kirchhoff surface include the instantaneous pressure, the pressure-time derivative, and the pressure gradient normal to the surface. The Kirchhoff code then computes the OASPL and the pressure-time signals in the near- and the far-field. From the pressure-time signals, it is possible to compute the SPL spectrum at any location with the aid of the Fast Fourier Transform (FFT).

\section{Results and Discussion}

\subsection{Jet Mean Flowfield}

Figure 3 shows the computed mean velocity contours in the jet. The potential core and the mixing of the turbulent shear layer are clearly displayed. A comparison of the predicted centerline mean axial velocity with the data is presented in figure 4 . Both the data and the CFD solution suggest that the jet potential core extends to about 15 jet radii from the nozzle exit plane. The jet potential core may be defined as the axial distance where the jet centerline velocity remains at about $99 \%$ of the jet exhaust velocity. It is known that the major noise production region occurs near the end of the potential core, where the most highly amplified instability wave reaches its maximum growth (Seiner et al. ${ }^{14}$ )

In the velocity decay region past the core, the CFD solution predicts a slower growth relative to the measurements. This discrepancy is attributable to the accuracy of the Spalart-Allmaras one-equation turbulence model. Also, the effects of compressibility are not appropriately taken into account in this turbulence model, as it was originally developed for incompressible flows. Data on compressible shear layers indicate that compressibility tends to reduce the growth rate of the shear layers (Goebel and Dutton ${ }^{16}$ ) relative to the incompressible case, with an increase in the jet convective Mach number. For example, before the potential core closes, a jet convective Mach number $M_{c}$, which may be defined by (Freund et al. ${ }^{17}$ ) 


$$
M_{c}=\frac{M_{j} \sqrt{T_{j} / T_{\infty}}}{1+\sqrt{T_{j} / T_{\infty}}}
$$

The reduced transverse turbulence intensity is believed to be responsible for the observed reduction in the spreading rate for compressible mixing layer. By taking into account the effects of compressibility on the turbulent kinetic energy and its specific dissipation rate into a twoequation turbulence model, the reduction in the mixing length growth rate is found to agree with the data (Kandula and Wilcox ${ }^{18}$ ).

Figures 5 and 6 respectively display the mean static pressure and mean Mach number along the centerline of the jet. The pressure oscillation along the centerline is relatively small and shows the accuracy of the boundary conditions. The centerline Mach number variation follows the trend of the centerline velocity, as is to be expected.

Calculations show that with an increase in jet temperature, the potential core length decreases. These results are in qualitative agreement with the data of Seiner et al. ${ }^{14}$. As the jet temperature increases, the convective Mach number increases, and the Mach wave radiation pattern is modified, with the OASPL values generally increasing with an increase in jet temperature.

\subsection{Jet Instantaneous Pressure and OASPL Contours}

Figure 7 displays the instantaneous pressure contours in the source-field, showing the directivity of Mach wave emission. The lack of significant reflection near the boundaries indicates that the outflow and the radiation boundary conditions implemented in the CFD code are satisfactory. A display of the OASPL within the jet, characterizing the nonlinear source-field, as directly computed by the CFD code, is presented in figure 8 . Inside the jet shear layer, the pressure levels are high (as much as $180 \mathrm{~dB}$ ), with the highest OASPL occurring about 7 jet radii downstream of the exit.

\subsection{Near-and-Farfield Acoustic Solutions}

Figure 9 presents the OASPL contours in the nearand the far-field as computed by the $\mathrm{CFD} /$ Kirchhoff formulation. It is seen that the OASPL ranges from $80 \mathrm{~dB}$ to $130 \mathrm{~dB}$ in this region. The directivity of the Mach wave radiation (emission) is evident. It is generally accepted that the mechanism for Mach wave radiation is strongly connected with the amplification of instability modes, and this amplification scales with the jet convective Mach number.

The variation of the OASPL in the streamwise direction at $z / r_{j}=80$ is compared in figure 10 with the measurements of Seiner et al.14. The predicted peak angle of emission of about 128 degrees is in good agreement with the data, which indicates a peak at 127 degrees. Also, the peak value of the OASPL is about $7 \mathrm{~dB}$ less than the measured value. There is an underprediction of the OASPL at the off-peak locations both upstream and downstream of the peak. This underprediction is primarily due to the axisymmetric simulations (axisymmetric disturbance mode $m=0$ instability) of the present investigation. It is impossible to develop a threedimensional helical mode instability with axisymmetric simulations. Measurements show that near the nozzle exit, axisymmetric modes seem to be dominant, and farther downstream the helical nature of the large-scale structure is dominant (Viswanathan and Sankar ${ }^{19}$ ). Also, both experiments and hydrodynamic stability analysis suggest that the $m=+1$ helical mode is dominant above a jet Mach number of about 1.4. Thus by including azimuthal mode $(m=+1)$ instability in a threedimensional analysis, predictions of sound pressure levels could be considerably improved (Gamet and Estivalezes ${ }^{20}$ ). Such three-dimensional simulations require considerably more computing resources, which are outside the scope of the present work. Further improvements in the predictions can be realized by considering inflow disturbances of various frequencies (or random disturbances).

Figure 11 shows the corresponding comparison with the OASPL plotted as a function of the axial distance from the exit plane. The computed results of OASPL for three values of $z / r_{j}=30,55$ and 80 are displayed in figure 12 , the last one corresponding to the data by Seiner ${ }^{14}$. With increasing values of $z / r_{j}$, the peak value of OASPL decreases, and the corresponding peak angle decreases. This is to be expected in view of the directivity of Mach wave radiation. The correctness of this trend also serves to verify the Kirchhoff code implementation.

The spectral content of SPL $(\mathrm{dB} / \mathrm{Hz})$ along a direction corresponding to the maximum radiation $\left(x / r_{j}=60, z / r_{j}=80\right)$ is displayed in figure 13. Figure 14 indicates the variation of $1 / 3$ octave SPL (dB) as a function of the frequency. A well-defined peak SPL value of about $128 \mathrm{~dB}$ is seen to occur at a frequency of about 2 kilohertz $(\mathrm{kHz})$, which corresponds to a Strouhal number of 0.2 . This value is consistent with the frequency of the imposed disturbance at the nozzle exit. Figure 15 presents the result of figure 14 in a semi-log plot.

\section{Conclusions}

The CFD/Kirchhoff approach is capable of predicting the SPL spectrum and the OASPL at any location in the near-field and the far-field. The relative reduction in the 
computational time due to the Kirchhoff method and the axisymmetric simulation make it an attractive engineering tool. The one-equation turbulence model of SpalartAllmaras for computing turbulent stresses affords additional reduction in the computational time and at the same time improves the robustness of the code relative to two-equation turbulence models. The peak angle of Mach wave radiation and the peak level of OASPL are satisfactorily predicted. Predictions at off-peak conditions could be substantially improved by considering three-dimensional disturbances (azimuthal mode instability) and disturbances of various frequencies.

\section{Acknowledgements}

The authors wish to thank Dr. Pieter Buning of NASA Langley Research Center for discussions with regard to the OVERFLOW code. Professor Anastasios Lyrintzis of Purdue University (School of Aeronautics and Astronautics) kindly provided the YORICK code for Kirchhoff integration. Thanks are also due to Dr. Ravi Margasahayam of Dynacs Inc., and Professor Michael Norton of the University of Western Australia for useful discussions. Detailed review of the report by Stanley Starr, Chief Engineer, Dynacs Inc. is gratefully acknowledged.

\section{References}

1. Lighthill, M.J., On Sound Generated Aerodynamically: I. General Theory, Proc. Roy. Soc. (London), Ser. A, Vol. 211, No. 1107, pp. 564-587, March 1952.

2. Lighthill, M.J., On Sound Generated Aerodynamically: II. Turbulence as a Source of Sound, Proc. Roy. Soc. (London), Ser. A, Vol. 222, No. 1148, pp. 1-32, Feb. 1954.

3. Tam, C.K.W., Computational Aeroacoustics; Issues and Methods, AIAA J., Vol. 33, No. 10, pp. 1788-1796, 1995.

4. Tam, C.K.W., and Webb, J.C., Dispersion-RelationPreserving Finite Difference Schemes for Computational Aeroacoustics, Journal of Computational Physics, Vol. 107, pp. 262-281, 1993.

5. Lele, S.K., Compact Finite Difference Schemes with Spectral-Like Resolution, J. of Computational Physics, Vol. 103, pp. 16-42, 1992.

6. Kirchhoff, G. R., Zur Theorie der Lichtstrahlen, Annalen der Physik und Chermie, Vol. 18, pp. 663-695, 1883.

7. Buning, P.G., Jesperson, D.C., Pulliam, T.H., Chan, W.M., Slotnick, J.P., Krist, S.E., and Renze, K.J., OVERFLOW User's Manual- version 1.8, NASA Langley Research Center, February 1998.
8. Spalart, P.R., and Allmaras, S.R., A One-Equation Turbulence Model for Aerodynamic Flows, AIAA-920439, 1992.

9. Thompson, K.W., Time-Dependent Boundary Conditions for Hyperbolic Systems II, Journal of Computational Physics, Vol. 89, pp. 439-461, 1990.

10. Hixon, R., Shih, S.-H, and Mankbadi, R., Evaluation of Boundary Conditions for Computational Aeroacoustics, AIAA J., Vol. 33, No. 11, pp. 2006-2012, November 1995.

11. Pilon, A.R., and Lyrintzis, A.S., Development of an Improved Kirchhoff Method for Jet Aeroacoustics, AIAA J., pp. 783-790, May 1998.

12. Morino, L., and Tseng, K., A General Theory of Unsteady Compressible Potential Flows with Applications to Airplanes and Rotors, in Developments in Boundary Element Methods, edited by P.K. Banerjee and L. Morino, Vol. 6, pp. 183-245, Elsevier Applied Science, Barking, England, UK, 1990.

13. Lyrintzis, A.S., and Mankbadi, R.R., Prediction of the Far-Field Jet Noise Using Kirchhoff's Formulation, AIAA J., Vol. 32, No.2, 413-416, February 1996.

14. Seiner, J.M., Ponton, M.K., Jansen, B.J., and Lagen, N.T., The Effects of Temperature on Supersonic Jet Noise Emission, AIAA 92-02-046, 1992.

15. Mankbadi, R., Hixon, H., Shih, S.-H., and Povinelli, L., Use of Linearized Euler Equations for Supersonic Jet Noise Prediction, AIAA J., Vol. 36, No.2, February 1998.

16. Goebel, S.G., and Dutton, J.C., Experimental Study of Compressible Turbulent Mixing Layers, AIAA J., Vol. 29, No. 4, pp. 453-477, 1991.

17. Freund, J.B., Lele, S.K., and Moin, P., Numerical Simulation of a Mach 1.92 Turbulent Jet and Sound Field, AIAA J., Vol. 38, No. 11, November 2000.

18. Kandula, M., and Wilcox, D.C., An Examination of $k-\omega$ Turbulence Model for Boundary Layers, Free Shear Layers, and Separated Flows, AIAA 95-2317, 1995.

19. Viswanathan, K., and Sankar, L.N., Toward the Direct Calculation of Noise: Fluid/Acoustic Coupled Simulation, Vol. 33, No. 12, pp. 2271-2279, 1995.

20. Gamet, L., and Estivalezes, J.L., Application of Large-Eddy Simulations and Kirchhoff Method to Jet Noise Prediction, AIAA J., Vol. 36, No. 12, pp. 21702178, December 1998. 
Table 1. Summary of Nozzle Conditions

\begin{tabular}{|l|l|}
\hline Stagnation temperature, $\mathrm{K}$ & 755 \\
\hline Nozzle exit diameter, $\mathrm{m}$ & 0.09144 \\
\hline Exit pressure, atm & 1.0 \\
\hline Exit temperature, $\mathrm{K}$ & 419 \\
\hline Exit velocity, m/s & 822 \\
\hline Acoustic velocity at exit, $\mathrm{m} / \mathrm{s}$ & 411 \\
\hline Nozzle exit Mach number & 2.0 \\
\hline Exit jet Reynolds number & $1.3 \times 10^{6}$ \\
\hline Ambient pressure, atm & 1.0 \\
\hline Ambient temperature, $\mathrm{K}$ & 280 \\
\hline
\end{tabular}

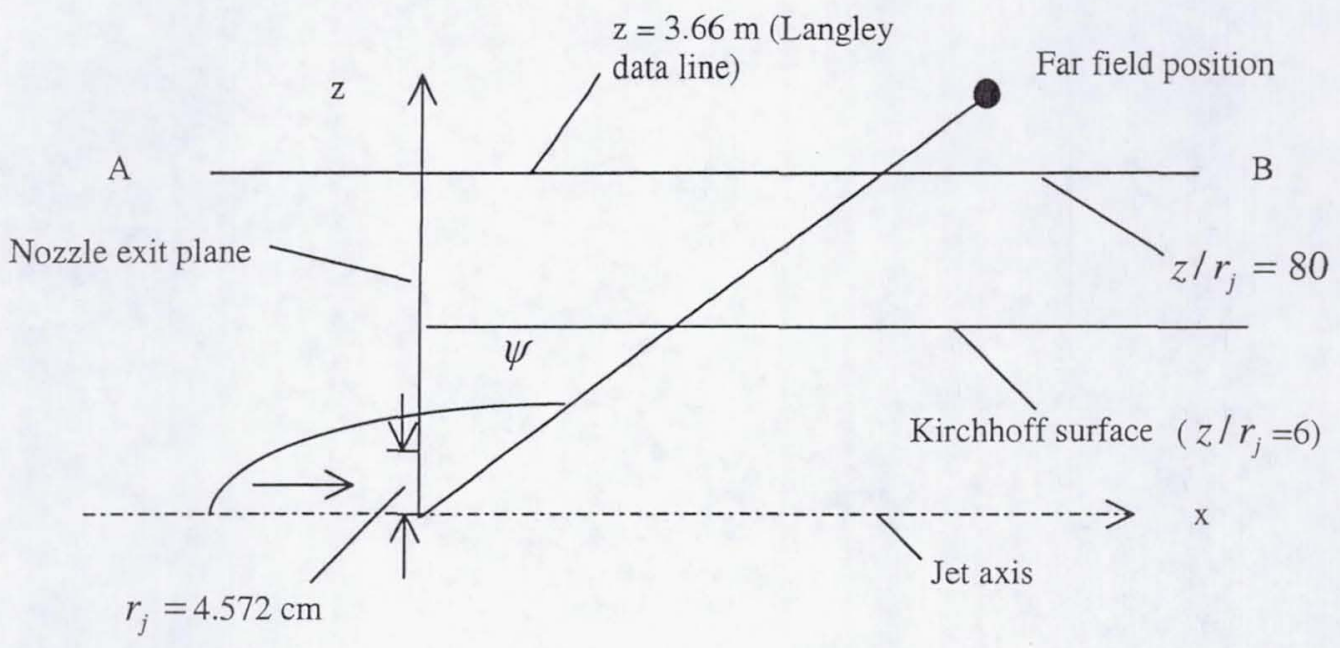

Figure 1. Schematic of the Test Case Nozzle

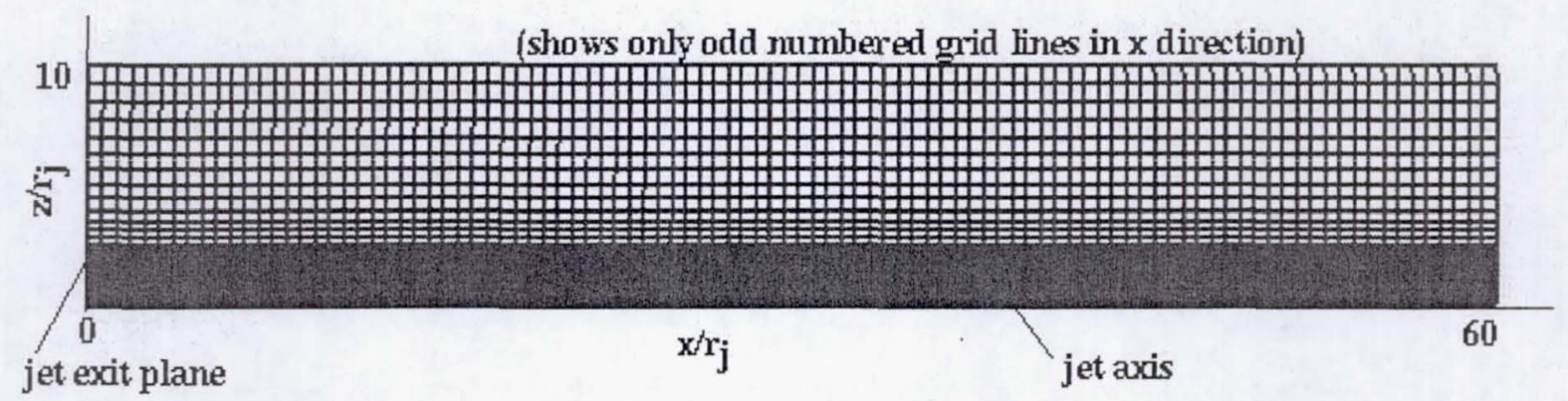

Figure 2. Axisymmetric grid (200 x 100) 


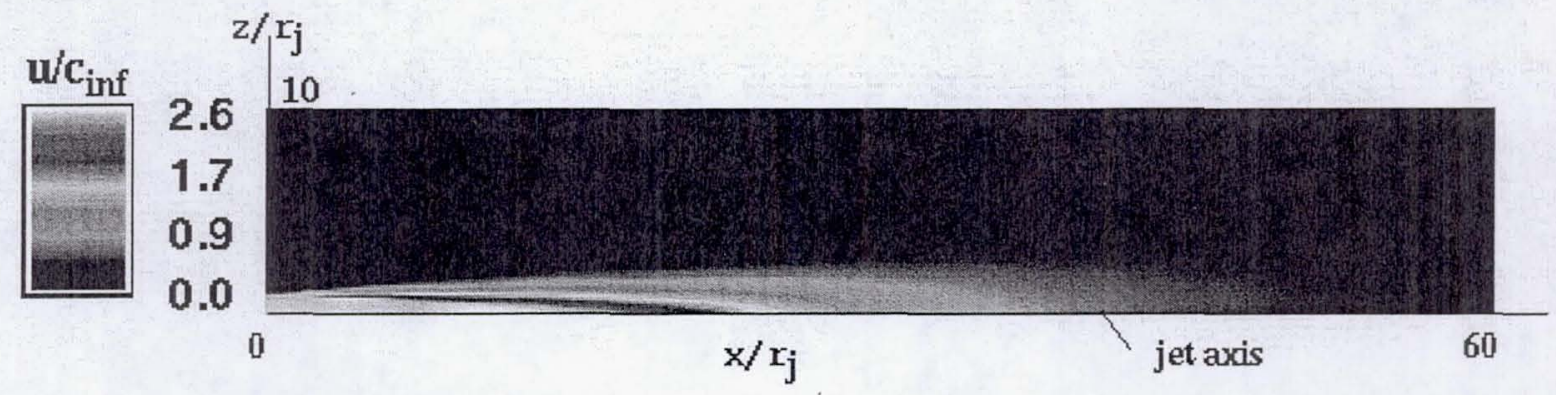

Figure 3. Mean Axial Velocity Contours in the Jet From CFD Solution

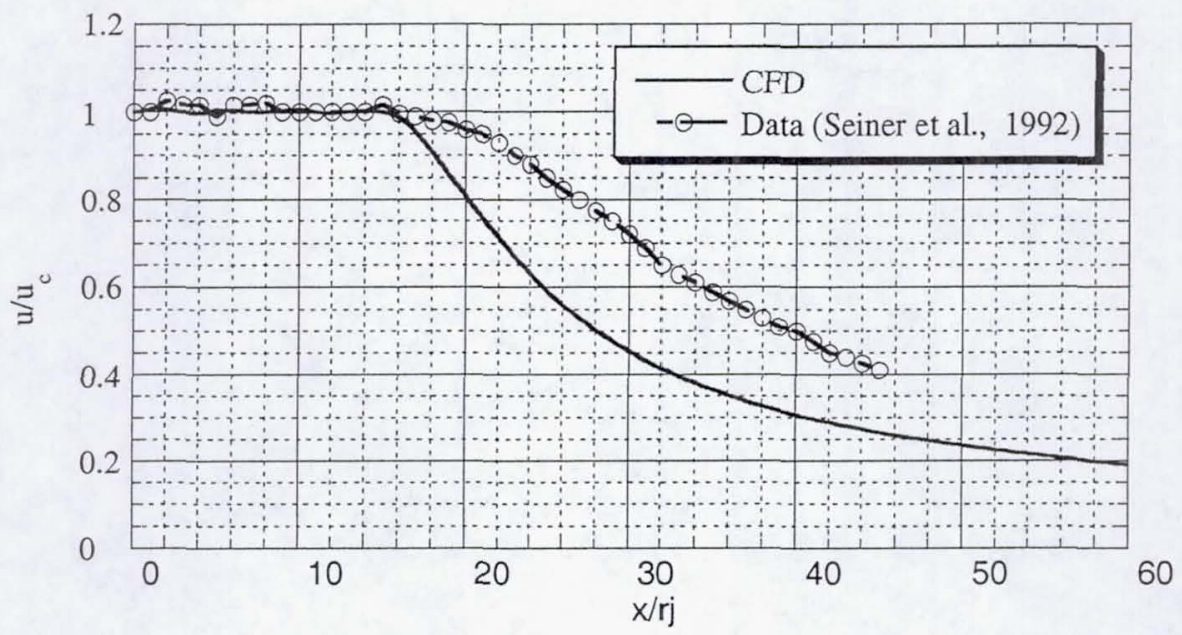

Figure 4. Jet Centerline Variation of Axial Velocity From CFD 


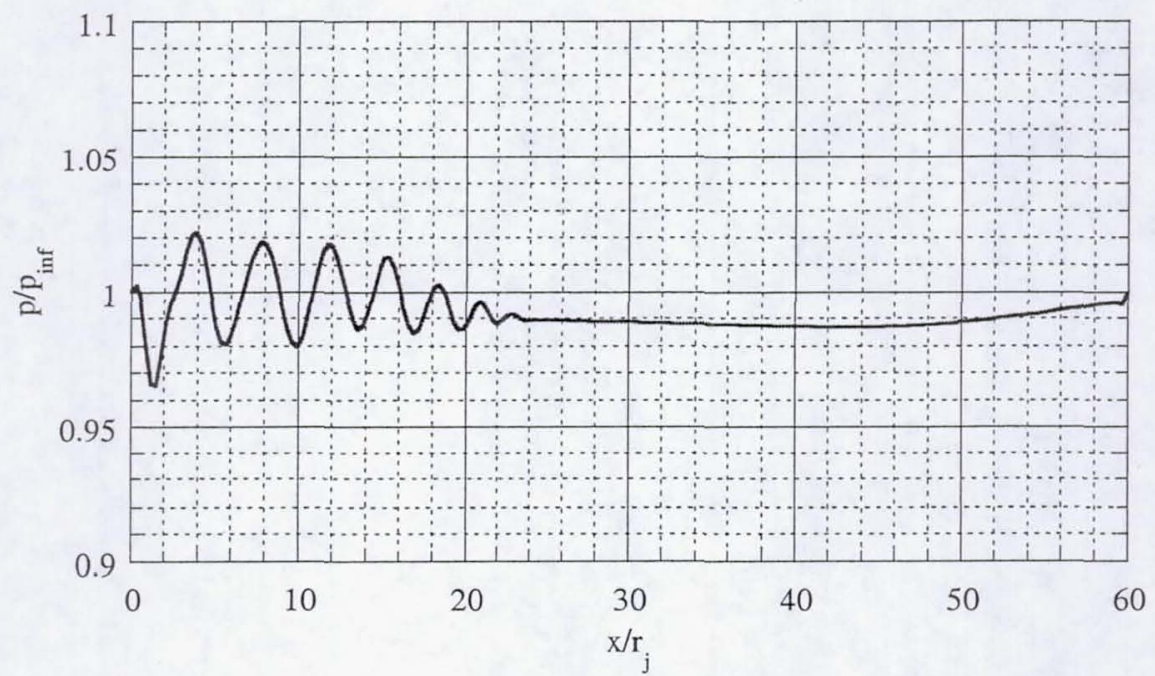

Figure 5. Jet Centerline Variation of Static Pressure From CFD

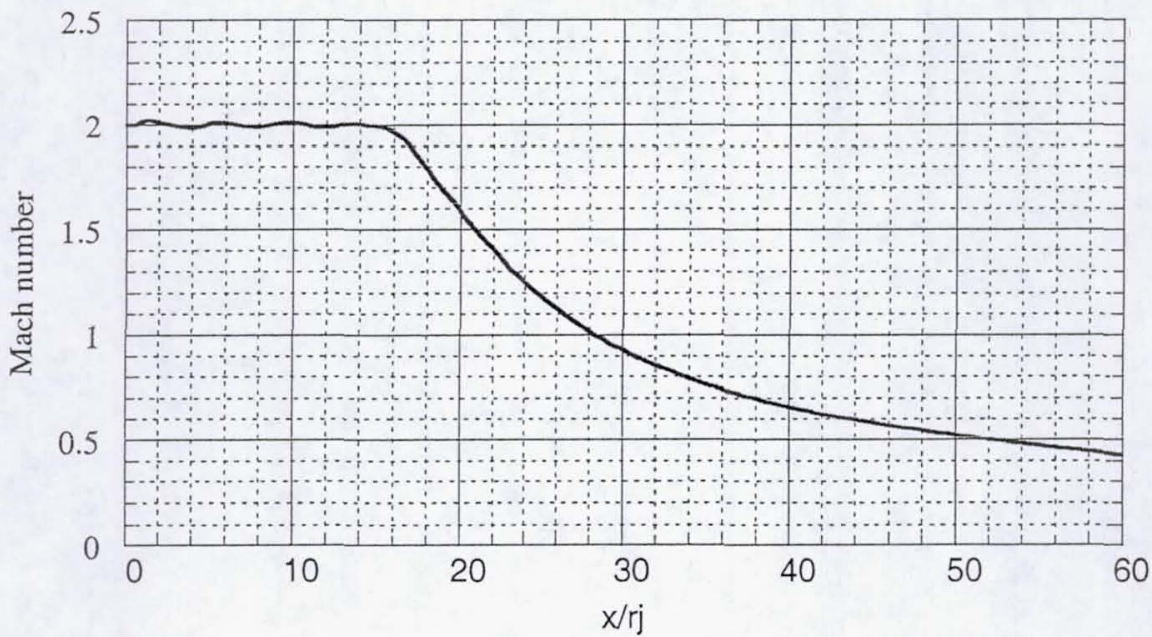

Figure 6. Jet Centerline Variation of Mach Number From CFD 


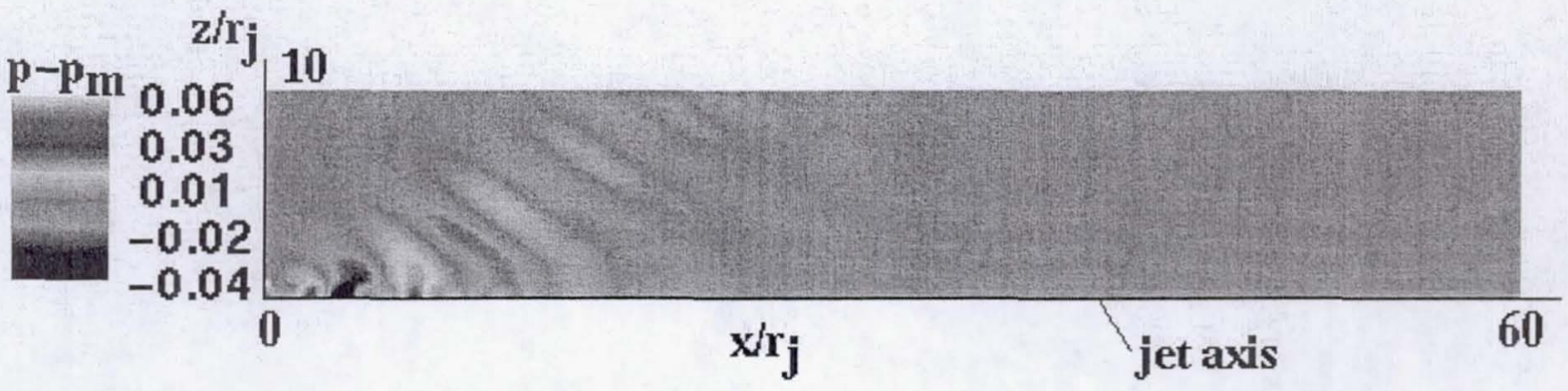

Figure 7. Instantaneous Pressure Contours in the Jet From CFD Solution

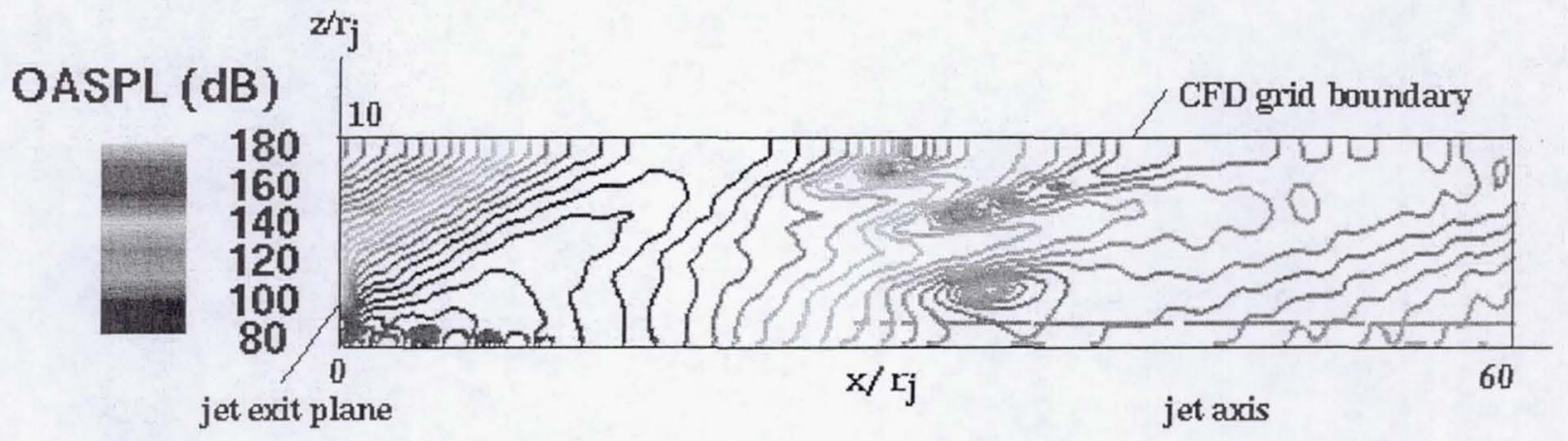

Figure 8. Contours of OASPL in the Source-Field as Computed Directly From CFD 


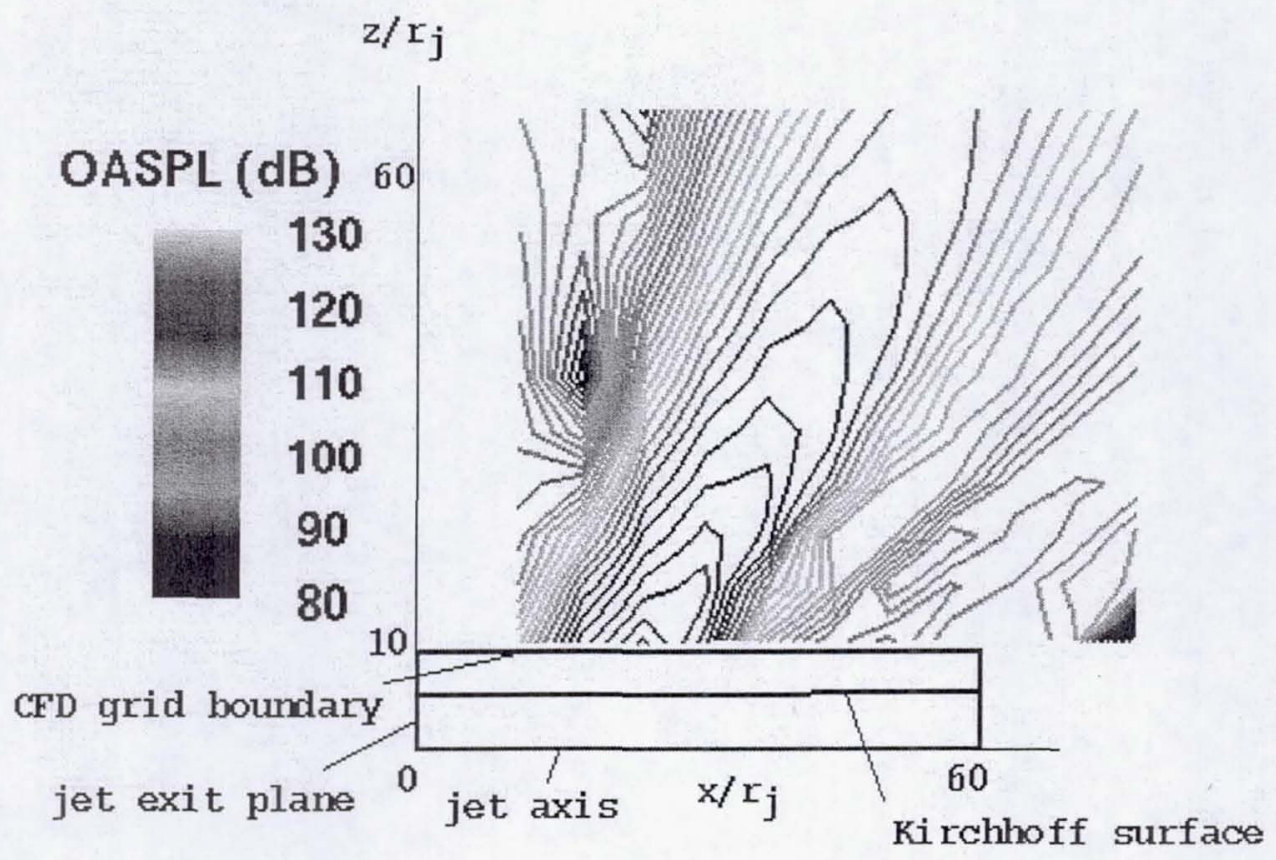

Figure 9. Contours of OASPL Predicted From CFD/Kirchhoff Method

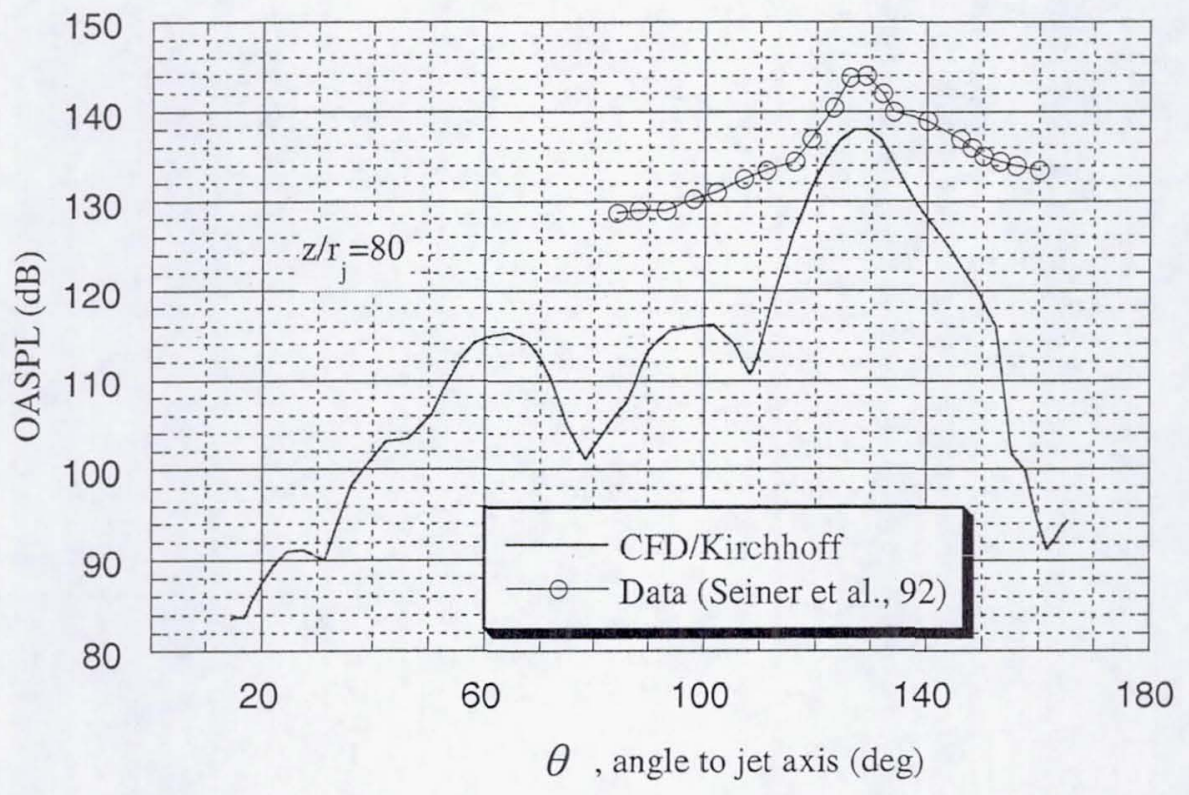

Figure 10. Comparison of Angular OASPL Distribution at $z / r_{j}=80$ 


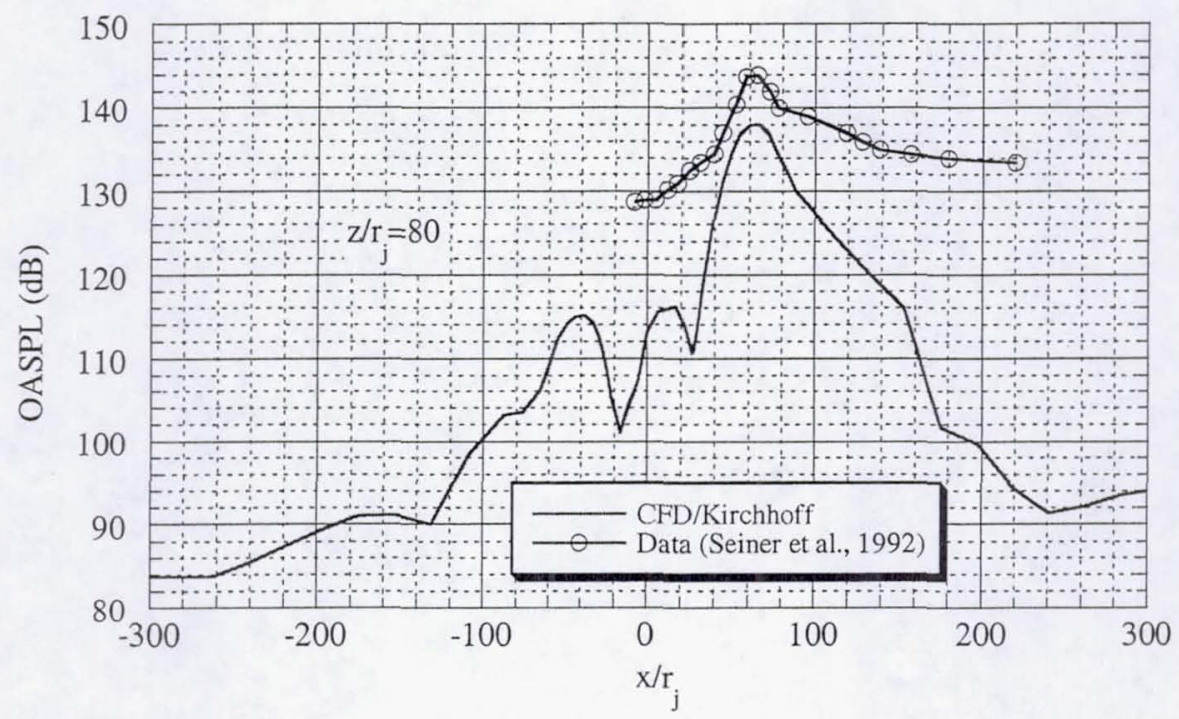

Figure 11. Comparison of Axial OASPL Distribution at $z / r_{j}=80$

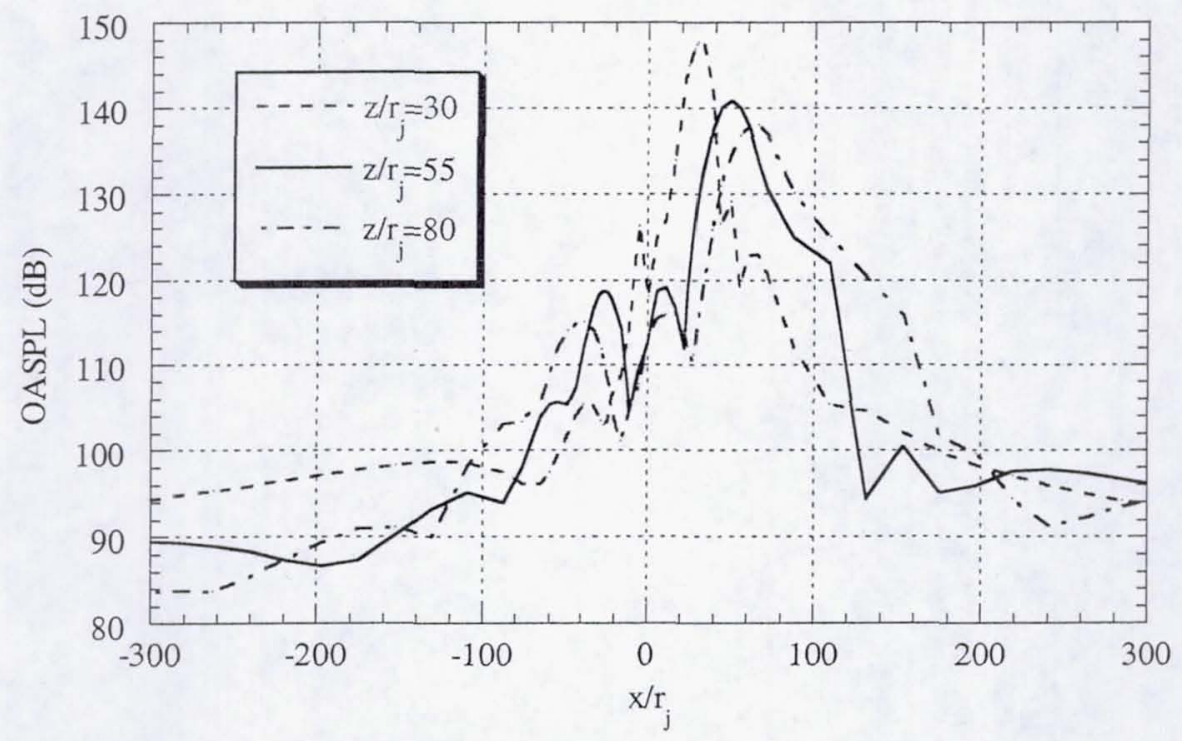

Figure 12. Computed Axial Distributions of OASPL for $z / r_{j}=30,55$, and 80 


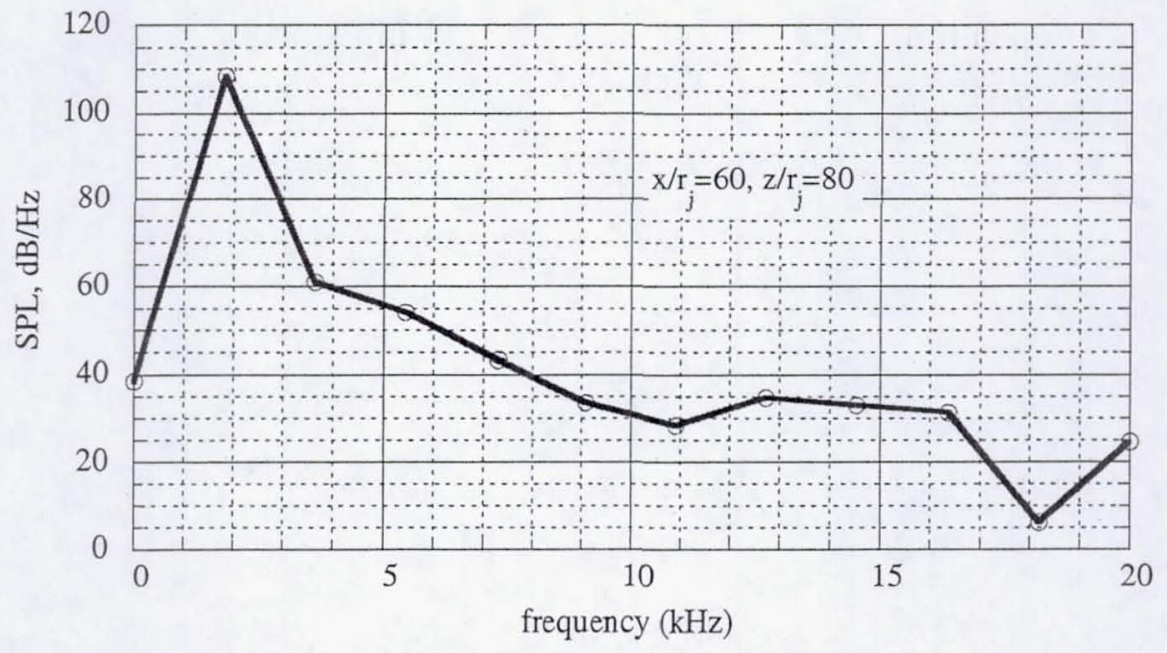

Figure 13. Computed SPL Distribution at $x / r_{j}=60$, and $z / r_{j}=80$

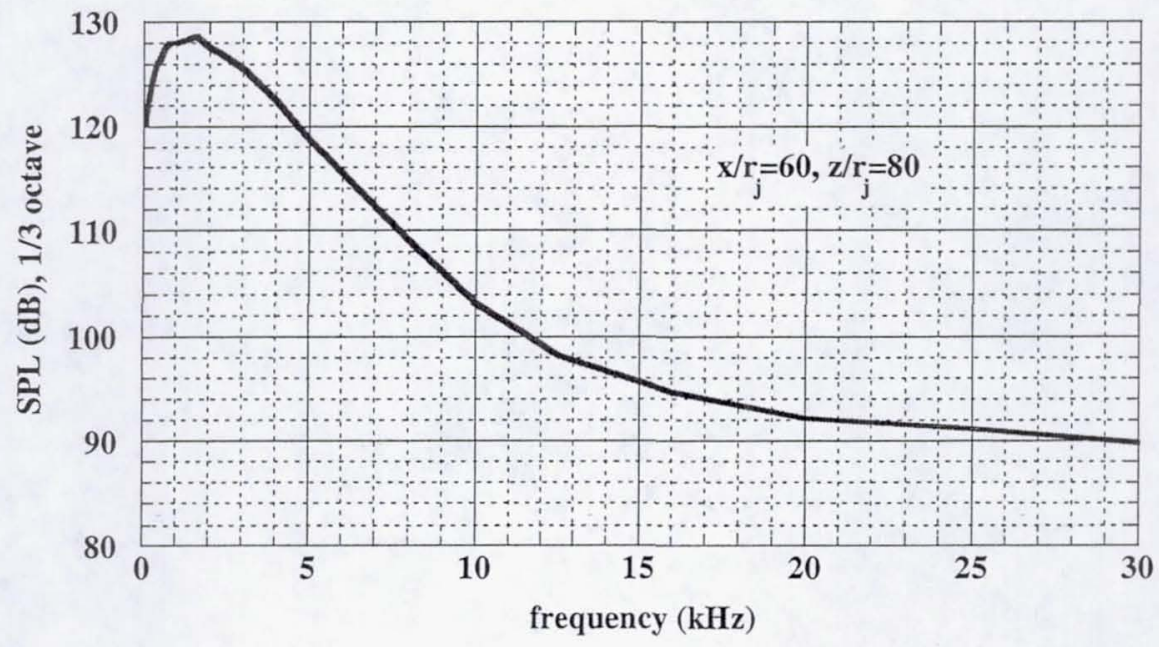

Figure 14. Computed SPL Distribution at $x / r_{j}=60$, and $z / r_{j}=80$ 


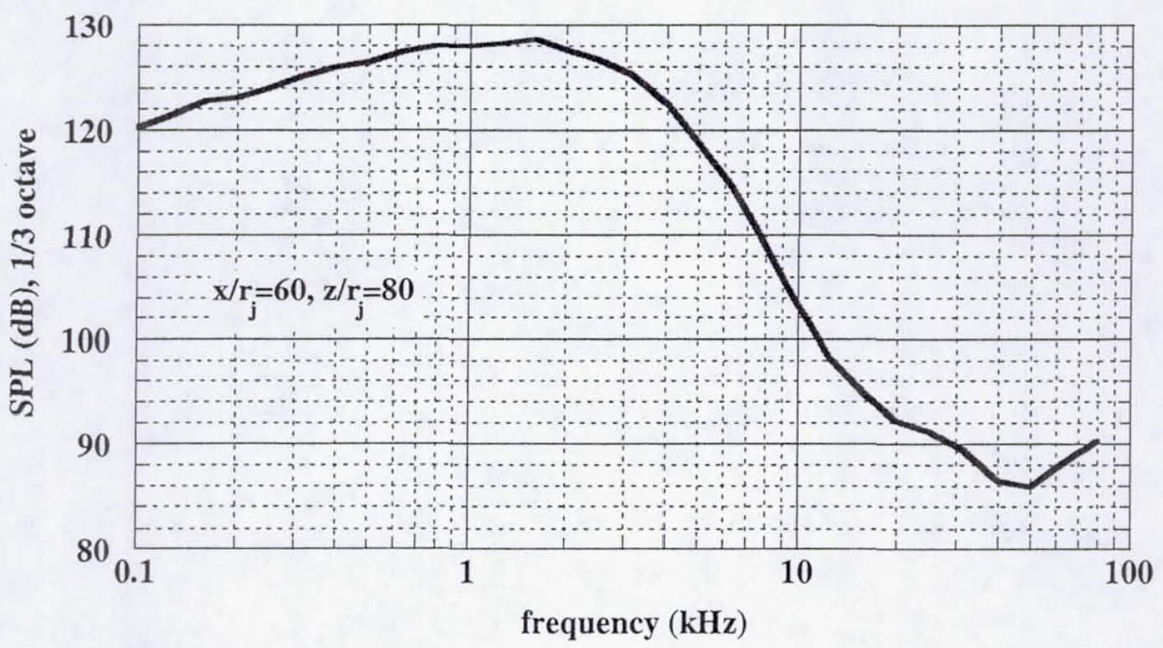

Figure 15. Computed SPL Distribution (Semi-Log Plot) at $x / r_{j}=60$, and $z / r_{j}=80$ 